\title{
Transcranial magnetic stimulation for schizophrenia
}

\author{
Nadine Dougall ${ }^{1}$, Andrew McIntosh ${ }^{2}$, and Klaus $\mathbf{P}$ Ebmeier ${ }^{3}$ \\ ${ }^{1}$ Division of Psychiatry, University of Edinburgh, Edinburgh, UK \\ 2University of Edinburgh, Royal Edinburgh Hospital, Edinburgh, UK \\ ${ }^{3}$ University of Edinburgh, Edinburgh, UK
}

\begin{abstract}
This is the protocol for a review and there is no abstract. The objectives are as follows:

To estimate the effects of TMS alone compared with sham TMS or with 'standard management' and any other comparison interventions in reducing psychotic symptoms associated with schizophrenia.
\end{abstract}

\section{BACKGROUND}

People with schizophrenia typically experience auditory hallucinations (hearing voices) or delusions (false beliefs) during acute episodes. Although several effective treatments are available, many patients have intractable symptoms that do not recover between acute episodes. In addition, motivation and social behaviour may also be adversely affected (negative symptoms). Relatively high numbers of people with schizophrenia have persistent symptoms in spite of apparently adequate drug treatment. In some cases treatment failure is associated with non-adherence, although it is understood that many people have enduring symptoms in spite of adequate treatment. Transcranial magnetic stimulation (TMS) could prove an alternative treatment for patients who do not cope well with standard medication.

Transcranial magnetic stimulation is a relatively new sophisticated device-based therapy which involves the skilful application of a strong magnetic field close to the surface of the scalp. The procedure is a non-invasive and relatively painless technique for stimulating the cerebral cortex and altering neuronal function (Chouinard 2003). The device uses specifically designed insulated wire coils which deliver strong and very brief magnetic pulses, passing from carefully chosen surface landmarks without hindrance into underlying

Copyright (C) 2009 The Cochrane Collaboration. Published by John Wiley \& Sons, Ltd.

Contact address: Nadine Dougall, Division of Psychiatry, University of Edinburgh, Royal Edinburgh Hospital, Kennedy Tower,

Edinburgh, EH10 5HF, UK. Nadine.dougall@ed.ac.uk.

Editorial group: Cochrane Schizophrenia Group.

Publication status and date: Edited (no change to conclusions), published in Issue 1, 2009.

CONTRIBUTIONS OF AUTHORS

Nadine Dougall - helped write protocol.

Andrew McIntosh - read abstracts, study selection and wrote review.

Klaus Ebmeier-resolved conflicts between authors, data extraction and wrote review

DECLARATIONS OF INTEREST

KPE - Loan of Dantec Magnetic Stimulator for 1 year; travel expenses paid by The Magstim Ltd; Research grant funded by MRCUK in collaboration with The Magstim Ltd. 
brain regions. The magnetic field then induces small transient electrical currents in the neural circuitry of treated individuals. By varying the intensity, duration and frequency of the magnetic field, the neuronal systems may be excited or inhibited for as long as the current pulse in the coil (Barker 2002).

\section{Technical background}

Brain activity has been shown to differ in people with schizophrenia compared to the brain activity of people who do have this condition. Whereas activity in the temporoparietal cortex (TPC) appears to increase in patients with schizophrenia experiencing auditory hallucinations (Shergill 2000), activity in the dorsolateral prefrontal cortex (DLPFC) appears to be reduced in people with schizophrenia (Weinberger 1996). Reduced activity also appears to be correlated with negative symptoms (e.g. decreased motivation and social function) experienced by the patient. It is possible that by normalising activity in these brain regions, auditory hallucinations and negative symptoms would also improve.

Transcranial Magnetic Stimulation has been applied in several trials in two main paradigms: high frequency TMS and low frequency TMS. Low frequency TMS $(1 \mathrm{~Hz})$ is typically applied to the left TPC of patients to hopefully decrease brain activity and reduce auditory hallucinations. High-frequency TMS is applied to left DLPFC in an attempt to increase activity and reduce negative symptoms. Low frequency TMS is considered to inhibit cortical activity (Chen 1997) and high frequency TMS generally increases cortical activity in stimulated areas (Pascual-Leone 1998). Positive (Hoffman 2005) and negative (McIntosh 2004) controlled studies have been published using both treatment approaches and it is unclear whether TMS represents a significant treatment advance.

In schizophrenia, there is evidence of both decreased and increased cortical activity compared to unaffected controls and in some cases the altered activity correlates with the presence of a known symptom of cognitive deficit. Studies have demonstrated an association between temporal lobe activity and auditory hallucinations in patients with schizophrenia (Hoffman 2000, d'Alfonso 2002, Lee 2005, Poulet 2005). Active stimulation has been found to significantly reduce hallucinations in comparison to sham stimulation (Hoffman 2000). Not all attempts at replication have unequivocally supported Hoffman's findings (McIntosh 2004, Saba 2005).

Placebo arms of trials of TMS often use sham treatments. There are limitations to this approach; no satisfactory placebo condition has been established and individuals may not have identical expectations of real or sham TMS. Placebo or sham TMS should result in scalp and noise sensation identical to active TMS, without the cortical stimulation. Although noise sensation can be mimicked, generating the scalp sensation may also produce a therapeutic cortical stimulation. Avoiding the confounding of cortical stimulation with sham TMS yields a control arm of the trial which typically controls for noise sensation but not for scalp sensation. In trials which implement sham TMS double blinding of observer and patient is not guaranteed and estimated efficacy rates of TMS will possibly be confounded if participants are aware which treatment arm they are in. 
Bearing in mind the limitations of the trial methodology and in the absence of an entirely inactive sham condition that mimics real TMS, this systematic review aims to evaluate the current evidence base of TMS in the treatment for schizophrenia. We wish to ascertain the efficacy and safety of TMS, explore sources of heterogeneity that might explain contradicting positive and negative effects, investigate whether pooled effect sizes can be derived and whether they are statistically robust and lastly provide recommendations where possible for future research.

\section{OBJECTIVES}

To estimate the effects of TMS alone compared with sham TMS or with 'standard management' and any other comparison interventions in reducing psychotic symptoms associated with schizophrenia.

\section{METHODS}

\section{Criteria for considering studies for this review}

Types of studies-We included all relevant randomised controlled trials with group sizes of at least five. We excluded quasi-randomised studies, such as those allocating by using alternate days of the week.

Types of participants-People with schizophrenia and related affective psychoses, diagnosed according to standardised operational criteria, irrespective of age and sex.

\section{Types of interventions}

1. Transcranial magnetic stimulation: at any stimulus voltage, frequency or charge, administered to the head at any location.

2. Sham TMS: TMS administered using fake instruments or with the coil applied at an oblique angle, greater than or equal to 45 degrees) to the skull.

3. Standard treatment: any treatment provided as part of routine care, however defined.

4. Any other pharmacological or non-pharmacological treatments given as part of an experimental intervention. Examples might include Electroconvulsive Therapy (ECT) and Cognitive Behaviour Therapy (CBT).

Types of outcome measures-We classified outcomes in to the seven categories detailed below. We divided outcomes into immediate (within two hours), short term (greater than two hours and up to and up to 24 hours) and medium term (greater than 24 hours and up to two weeks).

\section{Primary outcomes}

1. Hospital and service outcome

$$
\text { 1.1 Duration of hospital stay }
$$


1.2 Changes in hospital status (for example, changes from informal care to formal detention in care, changes of level of observation by ward staff and use of secluded nursing environment)

2. Satisfaction with care

2.1 Recipient of care

2.2 Informal care givers

\section{Secondary outcomes}

1. Global state

1.1 No clinically important change in global state (as defined by individual studies)

1.2 Mean endpoint global state score

1.3 Mean change in global state scores

2. Mental state

2.1 No clinically important change in general mental state (as defined by individual studies)

2.2 Mean endpoint general mental state score

2.3 Mean change in general mental state scores

2.4 No clinically important change in specific symptoms

2.5 Mean endpoint specific symptom score

2.6 Mean change in specific symptom scores

3. Cognitive state

3.1 No clinically important change in cognitive state (as defined by individual studies)

3.2 Mean endpoint cognitive state score

3.3 Mean change in cognitive state scores

3.4 Mean endpoint specific cognitive state score

3.5 Mean change in specific cognitive state scores

4. Adverse effects

4.1 Incidence of adverse effects, general or specific

4.2 Leaving the study early

4.3 Measured acceptance of treatment

4.4 Use of antiparkinsonian treatment

4.5 Sudden and unexpected death 
5. Hospital and service outcome

5.1 Hospitalisation of people in the community

5.2 Severity of symptoms when dismissed from hospital

5.3 Changes in services provided by community teams

6. Satisfaction with care

6.1 Professional carers

7. Economic outcomes

\section{Search methods for identification of studies}

Electronic searches-The Cochrane Schizophrenia Group's Trials register (June 2006) using the phrase:

[( $(*$ TMS $*$ OR *transcranial $*$ OR *trans-cranial* OR *magnetic * $)$ in REFERENCE) and (magn* in STUDY)]

This register is compiled by systematic searches of major databases, hand searches and conference proceedings (see Group Module).

\section{Searching other resources}

1. Hand searching

We searched reference lists of included and excluded studies for additional relevant studies.

2. Requests for additional data

2.1 We contacted Magstim Company Ltd., the company who markets TMS machines in the UK, for published and unpublished data on the treatment (Table 1).

2.2 We contacted authors of relevant studies to enquire about other sources of relevant information.

\section{Data collection and analysis}

1. Study selection

1.1 ND and AM independently inspected citations identified by the search strategy and selected those citations thought to be relevant.

1.2 ND and KE independently read the abstracts of the citations selected in 1.1 and refined the list of potentially relevant articles for inclusion.

1.3 We ordered the full study articles identified in 1.2 and assessed these for inclusion/exclusion criteria and methodological quality. At all stages, we resolved disputes by discussion.

2. Assessment of quality 
We independently allocated trials to three quality categories, as described in the Cochrane Reviewers' Handbook (Higgins 2005). Where disputes arose regarding which category it was felt a trial belonged to, where possible we resolved this by discussion. Where this was not possible and further information was necessary to clarify into which category to allocate the trial, we did not enter data but allocated the trial to the list of those awaiting assessment. We only included trials in Categories A or B in the review.

3. Data management

\subsection{Data extraction}

We extracted data independently and where further clarification was needed we contacted the authors of trials to provide us with the missing data.

\subsection{Intention to treat analysis}

We excluded data from studies where more than $50 \%$ of participants in any group were lost to follow up (this does not include the outcome of 'leaving the study early'). In studies with less than 50\% dropout rate, we considered people leaving early to have had the negative outcome, except for the event of death. Regarding the outcomes of 'aggression', 'self harm' and 'harm to others', as they are major risks of non-treated acute psychotic illness, we considered 5\% of the people leaving the study early to have had a negative outcome.

We analysed the impact of including studies with high attrition rates (25-50\%) in a sensitivity analysis. If inclusion of data from this latter group did result in a substantive change in the estimate of effect, we did not add their data to trials with less attrition, but presented them separately.

4. Data analysis

\subsection{Binary data}

For binary outcomes we calculated a standard estimation of the random effects risk ratio (RR) and its 95\% confidence interval (CI). We also calculated the weighted number needed to treat statistic (NNT), and its 95\% confidence interval (CI). If heterogeneity was found (see section 5) we used a random effects model.

4.2 Continuous data

4.2.1 Skewed data: continuous data on clinical and social outcomes are often not normally distributed. To avoid the pitfall of applying parametric tests to nonparametric data, the following standards are applied to all data before inclusion: (a) standard deviations and means were reported in the paper or were obtainable 
from the authors; (b) when a scale started from the finite number zero, the standard deviation, when multiplied by two, was less than the mean (as otherwise the mean was unlikely to be an appropriate measure of the centre of the distribution (Altman 1996); (c) if a scale started from a positive value (such as PANSS which can have values from 30 to 210) the calculation described above was modified to take the scale starting point into account. In these cases skew is present if $2 \mathrm{SD}>(\mathrm{S}-\mathrm{Smin})$, where $\mathrm{S}$ is the mean score and Smin is the minimum score. Endpoint scores on scales often have a finite start and end point and these rules can be applied to them. When continuous data are presented on a scale which includes a possibility of negative values (such as change on a scale), there is no way of telling whether data are non-normally distributed (skewed) or not. It is thus preferable to use scale end point data, which typically cannot have negative values. If end point data were not available, we used change data, but did not subject the data to a meta-analysis, and reported the results in the 'Additional data' tables.

4.2.2 Summary statistic: for continuous outcomes we estimated a weighted mean difference (WMD) between groups. Again, if heterogeneity was found (see section 5) we used a random effects model.

4.2.3 Valid scales: we included continuous data from rating scales only if the measuring instrument had been described in a peerreviewed journal and the instrument was either a self report or completed by an independent rater or relative (not the therapist).

4.2.4 Endpoint versus change data: where possible we presented endpoint data. If both endpoint and change data were available for the same outcomes we reported only the former.

4.2.5 Cluster trials: studies increasingly employ 'cluster randomisation' (such as randomisation by clinician or practice) but analysis and pooling of clustered data poses problems. Firstly, authors often fail to account for intra class correlation in clustered studies, leading to a 'unit of analysis' error (Divine 1992) whereby $p$ values are spuriously low, confidence intervals unduly 
narrow and statistical significance overestimated. This causes type I errors (Bland 1997, Gulliford 1999).

Where clustering was not accounted for in primary studies, we presented the data in a table, with a $(*)$ symbol to indicate the presence of a probable unit of analysis error. In subsequent versions of this review we will seek to contact first authors of studies to obtain intra class correlation co-efficients of their clustered data and to adjust for this by using accepted methods (Gulliford 1999). Where clustering has been incorporated into the analysis of primary studies, we will also present these data as if from a noncluster randomised study, but adjusted for the clustering effect. We have sought statistical advice and have been advised that the binary data as presented in a report should be divided by a 'design effect'. This is calculated using the mean number of participants per cluster $(m)$ and the intra-class correlation co-efficient (ICC) Design effect $=1+(\mathrm{m}-1) * \mathrm{ICC}$ (Donner 2002). If the ICC was not reported it was assumed to be 0.1 (Ukoumunne 1999).

If cluster studies had been appropriately analysed taking into account intra-class correlation co-efficients and relevant data documented in the report, synthesis with other studies would have been possible using the generic inverse variance technique.

5. Investigation for heterogeneity

Firstly, we considered all the included studies within any comparison to judge clinical heterogeneity. Then we visually inspected graphs to investigate the possibility of statistical heterogeneity. This was supplemented, primarily, by employing the I-squared statistic. This provides an estimate of the percentage of inconsistency thought to be due to chance. Where the I-squared estimate was greater than or equal to $75 \%$, we interpreted this as evidence of high levels of heterogeneity (Higgins 2003). In any event of the I-squared estimate, the data were pooled using a random-effects model. Where inconsistency was substantial, i.e. greater than or equal to $75 \%$, we explored reasons for heterogeneity via a sensitivity analysis.

6. Addressing publication bias

We entered data from all included studies into a funnel graph (trial effect against trial size) in an attempt to investigate the likelihood of overt publication bias (Egger 1997).

7. Sensitivity analyses 
We analysed the effect of including studies with high attrition rates in a sensitivity analysis. Where a trial was described as 'double-blind' but implied that the study was randomised, we also included this in a sensitivity analysis.

8. General

Where possible, we entered data in such a way that the area to the left of the line of no effect indicated a favourable outcome for TMS.

\section{Acknowledgments}

We would like to thank the editorial base of the Cochrane Schizophrenia Group for their help. We gratefully acknowledge the financial support of the Gordon Small charitable trust.

\section{SOURCES OF SUPPORT}

Internal sources

- $\quad$ No sources of support supplied

External sources

- Gordon Small Charitable Trust for Research in Old Age Psychiatry, UK.

\section{WHAT'S NEW}

\begin{tabular}{lll}
\hline Date & Event & Description \\
\hline 28 October 2008 & Amended & Converted to new review format. \\
\hline
\end{tabular}

\section{HISTORY}

Protocol first published: Issue 3, 2006

\section{Additional references}

Altman 1996 . Altman DG, Bland JM. Statistics notes: detecting skewness from summary information. BMJ. 1996; 313:1200. [PubMed: 8916759]

Barker 2002 . Barker, AT. The history and basic principles of magnetic nerve stimulation. In: Pascual-leone, A.; Davey, N.; Rothwell, J.; Wassermann, EM.; Puri, BK., editors. Handbook of Transcranial Magnetic Stimulation. 1st Edition. Oxford University Press; New York: 2002. p. 3-17.

Bland 1997 . Bland JM. Statistics notes. Trials randomised in clusters. BMJ. 1997; 315:600. [PubMed: 9302962]

Chen 1997 . Chen R, Classen J, Gerloff C, Celnik P, Wassermann EM, Hallett M, Cohen LG. Depression of motor cortex excitability by low-frequency transcranial magnetic stimulation. Neurology. 1997; 48(5):1398-1403. [PubMed: 9153480]

Chouinard 2003 . Chouinard PA, Van der Werf YD, Leonard G, Paus T. Modulating neural networks with transcranial magnetic stimulation applied over the dorsal premotor and primary motor cortices. Journal of Neurophysiology. 2003; 90:1071-83. [PubMed: 12702714]

d'Alfonso 2002 . d'Alfonso AA, Aleman A, Kessels RP, Schouten EA, Postma A, van Der Linden JA, Cahn W, Greene Y, de Haan EH, Kahn RS. Transcranial magnetic stimulation of left auditory cortex in patients with schizophrenia: effects on hallucinations and neurocognition. Journal of Neuropsychiatry and Clinical Neuroscience. 2002; 14(1):77-9. 
Divine 1992 . Divine GW, Brown JT, Frazier LM. The unit of analysis error in studies about physicians' patient care behavior. Journal of General Internal Medicine. 1992; 7(6):623-9. [PubMed: 1453246]

Donner 2002 . Donner A, Klar N. Issues in the meta-analysis of cluster randomized trials. Statistics in Medicine. 2002; 21:2971-80. [PubMed: 12325113]

Egger 1997 . Egger M, Smith GD, Schneider M, Minder C. Bias in meta-analysis detected by a simple, graphical test. BMJ. 1997; 315:629-34. [PubMed: 9310563]

Gulliford 1999 . Gulliford MC. Components of variance and intraclass correlations for the design of community-based surveys and intervention studies: data from the Health Survey for England 1994. American Journal of Epidemiology. 1999; 149:876-83. [PubMed: 10221325]

Higgins 2003 . Higgins JPT, Thompson SG, Deeks JJ, Altman DG. Measuring inconsistency in meta-analyses. BMJ. 2003; 327:557-60. [PubMed: 12958120]

Higgins 2005 . Higgins, JPT.; Green, S. The Cochrane Library. John Wiley \& Sons, Ltd.; Chichester, UK: 2005. Cochrane Handbook for Systematic Reviews of Interventions 4.2.5 [updated May 2005].

Hoffman 2000 . Hoffman RE, Boutros NN, Hu S, Berman RM, Krystal JH, Charney DS. Transcranaial magnetic stimulation and auditory hallucinations in schizophrenia. Lancet. 2000; 355(9209):1073-75. [PubMed: 10744097]

Hoffman 2005 . Hoffman RE, Gueorguieva R, Hawkins KA, Varanko M, Boutros NN, Wu Y, Carroll K, Krystal JH. Temporoparietal Transcranial Magnetic Stimulation for Auditory Hallucinations: Safety, Efficacy and Moderators in a Fifty Patient Sample. Biological Psychiatry. 2005; 58:97-104. [PubMed: 15936729]

Lee 2005 . Lee S-H, Kim W, Chung Y-C, Jung K-H, Bahk W-M, Jun T-Y, Kim K-S, George MS, Chae J-H. A double blind study showing that two weeks of daily repetitive TMS over the left or right temporoparietal cortex reduces symptoms in patients with schizophrenia who are having treatment-refractory auditory hallucinations. Neuroscience Letters. 2005; 376:177-81. [PubMed: 15721217]

McIntosh 2004 . McIntosh AM, Semple D, Tasker K, Harrison LK, Owens DGC, Johnstone EC, Ebmeier KP. Transcranial magnetic stimulation for auditory hallucinations in schizophrenia. Psychiatry Research. 2004; 127:9-17. [PubMed: 15261700]

Pascual-Leone 1998 . Pascual-Leone A, Tormos JM, Keenan J, Tarazona F, Canete C, Catala MD. Study and modulation of human cortical excitability with transcranial magnetic stimulation. Journal of Clinical Neurophysiology. 1998; 15(4):333-34. [PubMed: 9736467]

Poulet 2005 . Poulet E, Brunelin J, Bediou B, Bation R, Forgeard L, Dalery J, d'Amato T, Saoud M. Slow transcranial magnetic stimulation can rapidly reduce resistant auditory hallucinations in schizophrenia. Biological Psychiatry. 2005; 57:188-91. [PubMed: 15652879]

Saba 2005 . Saba G, Verdon CM, Kalalou K, Rocamora JF, Dumortier G, Benadhira R, Stamatiadis L, Vicaut E, Lipski H, Januel D. Transcranial magnetic stimulation in the treatment of schizophrenic symptoms: A double blind sham controlled study. Journal of Psychiatric Research. 2005 in press:N/A.

Shergill 2000 . Shergill SS, Brammer MJ, Williams SC, Murray RM, McGuire PK. Mapping auditory hallucinations in schizophrenia using functional magnetic resonance imaging. Archives of General Psychiatry. 2000; 57:1033-38. [PubMed: 11074868]

Ukoumunne 1999 . Ukoumunne OC, Gulliford MC, Chinn S, Sterne JAC, Burney PGJ. Methods for evaluating area-wide and organistation-based intervention in health and health care: a systematic review. Health Technology Assessment. 1999; 3(5):1-75.

Weinberger 1996 . Weinberger DR, Berman KF. Prefrontal function in schizophrenia: confounds and controversies. Philosophical Transactions of the Royal Society of London. 1996; 351:1495-503. [PubMed: 8941961]

* Indicates the major publication for the study 


\section{Magstim Company Limited}

\section{Table 1}

\begin{tabular}{l} 
Contact details \\
\hline Mr John H Starzewski \\
Managing Director \\
Magstim Limited \\
Spring Gardens \\
Whitland SA34 0HR \\
UK \\
Telephone: +44 1994 241093 \\
URL: http://www.magstim.com/index.html
\end{tabular}

